\title{
Indore Navigation Mobile Application using Indore Positioning System (IPS)
}

\section{Anusha Sanampudi}

\begin{abstract}
Indoor Positioning system (IPS) is the technology that is used to locate smart phones, people or other objects inside a building where Global Positioning System (GPS) doesn't work or lack precision such as airports, underground locations, parking, multi-storey buildings etc...There is no fixed standard for implementing IPS rather it could be customized according to the location chosen. IPS in turn uses a number of technologies such as Wi-Fi, Bluetooth, Beacons, magnetic positioning, dead reckoning etc...Among the various technologies available studies prove that Magnetic localization provides a most efficient solution for Indoor positioning. Our paper focuses on building an indoor navigation mobile application for a retail store that allows users to search for a product and navigate them to the particular aisle in which the product is located. There by enabling the application to be location sensitive and context aware. In order to collect magnetic fingerprints and convert the obtained data into latitude and longitude values we make use of an API called IndoorAtlas, which helps in locating smart phones inside a building using the accelerometer, gyroscope, magnetometer and Bluetooth in a mobile. Magnetic localization is the concept where deflections of magnetic field from the steel structures inside the building will be captured by the magnetometer and other sensors within a mobile and that will be used to locate a smart phone inside a building. The same application could be utilized for various use cases such as Supermarkets \& Hypermarkets, museums \& galleries, Libraries, Hospitals, Airports \& stations, Shopping malls, Exhibition and Conferences.
\end{abstract}

Keywords : Beacons, IndoorAtlas, Indoor Navigation, Indoor Positioning System, Magnetic localization, Way Finding.

\section{INTRODUCTION}

Indoor Positioning and Navigation System is emerged as a solution for the raising navigation issue with the mobility of population in complex building layouts. Global Positioning system (GPS) is the most widely used satellite based technology for outdoor positioning and navigation. Satellite based technology cannot be used in indoor navigation because the structure of the indoor environment distorts or absorbs the signals (attenuation of signals). Implementation of Indoor Positioning System (IPS) becomes tedious as there are various obstacles in indoor such as walls, equipment, human beings, influencing the propagation of electromagnetic waves, which lead to multipath effects. Also some interference and noise sources from other wired and wireless networks degrade the accuracy of positioning in indoor environment.

Revised Manuscript Received on February 07, 2020.

* Correspondence Author

Anusha Sanampudi*, CSE, R.M.K Engineering College, Anna University, Chennai, India. Email: asp.cse@rmkec.ac.in

(C) The Authors. Published by Blue Eyes Intelligence Engineering and Sciences Publication (BEIESP). This is an open access article under the CC BY-NC-ND license (http://creativecommons.org/licenses/by-nc-nd/4.0/)
A number of approaches have been evolved for IPS, but none of them were deployable in most of the use cases in spite of their satisfactory accuracy in the experiments carried out. To develop an Indoor Navigation Systems that provides path for the user inside buildings,individual levels and signal losses due to various interferences has to be taken into account.IPS have several use cases, this paper deals with implementing an IPS for retail. The aim of the research is to build a user friendly android based Indoor Positioning and Navigation Application that should be able to generate route between the user's location and the known point of the destination using Magnetic localization and Bluetooth positioning techniques@.

\section{RELATED WORK}

In this era of technology, indoor positioning system has begun to develop and emerged as the most researched topic due to its number of end user applications. It is a system to determine the location of the objects or the people in a specified area by using radio waves, magnetic fields and other sensor signals obtained by computing devices. The Global Positioning System(GPS)can be used in outdoor environment to determine user position and provide path to the destination but when it comes to indoor environment, the effectiveness of the satellite based communication is affected by the multiple reflections suffered by the RF signal. The independent solutions that are used in past years are wireless technology, magnetic localization and BLE beacons. The major limitation provided by wireless technology is accuracy which is affected by user movements and obstacles and the power consumption in public areas. Both BLE and Wi-Fi cannot be used together as its signals cause interference and both the technologies need some sort of infrastructure. Magnetic localization is a technique which uses the magnetic fields emitted by the steels in the building to locate the software device(smart phones). Magnetic localization technique does not require any additional infrastructure and since most smart phones have sensors like magnetometer and compass, collecting magnetic fingerprints (magnetic fields) can be easily done. But this technique suffers from location accuracy. There is no benefitable research on any single technology. Hence, by coupling magnetic localization with beacons accuracy can be improved in Indoor Positioning System(IPS). Beacons are small hardware devices that emit Bluetooth Low Energy (BLE) signals. In recent years, beacons have become popular due to its inexpensive nature and consumption of less power. BLE beacon can be placed anywhere in the site due to its small dimension.

Published By: 
BLE beacons along with magnetic positioning generate better accuracy results than any other technology. This fusion technology provides an accuracy of 95\% while WIFI provides an accuracy of $75.83 \%$ (based on research).

\section{PROPOSED WORK}

An android application for indoor navigation in retail using magnetic localization (done using IndoorAtlas API) and BLE beacons (for accuracy), allows the user to search for products and navigate them to a particular aisle in which the product is located. Beacons are also used for providing promotional offers(Push Notification) related to search.

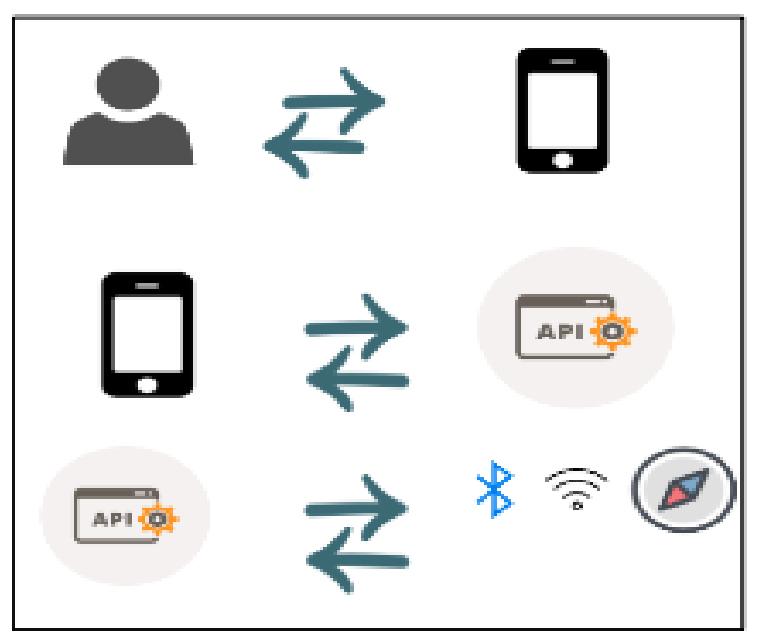

Fig. 1. Architecture

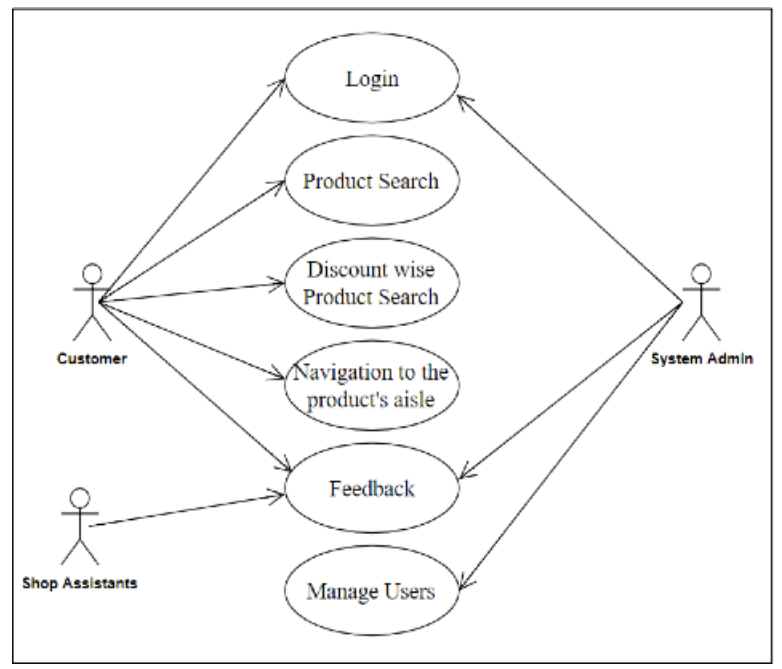

Fig. 2.Usecase Diagram

\section{A. System Overview}

IndoorAtlas's API provides a platform (ios / android application) which will be used for floor plan creation and managing the fingerprint collection and map generation. IndoorAtlas is used for localization purpose. It collects the magnetic fingerprints and also the BLE beacon RSSI (Received Signal Strength Identifier) nearby, for localization. Beacons are used to increase the positioning accuracy. Sensors like gyroscope, magnetometer, accelerometers are used by the IndoorAtlas's API for collecting the finger prints. After creating and uploading the floor plan (according to the dimensions of the retail) and collecting the fingerprints in the API, any android application can access the location (latitude and longitude values) by making a call to the IndoorAtlas API. The location will be rendered as an image view in the application which is mapped with the API.

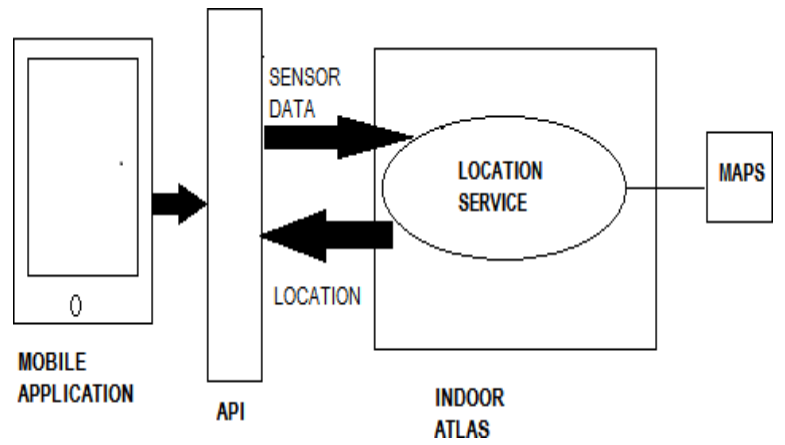

Fig. 3. System Interaction

\section{B. Methodology}

\section{A. Floor Plan Creation}

Focusing the corners of the store to get the 2D view of the floor (with dimensions of each aisle) thereby covering the entire site for generating the floor plan of the retail using the map box tool (IndoorAtlas). The floor plan of multilevel store can also be generated and labeled accordingly.

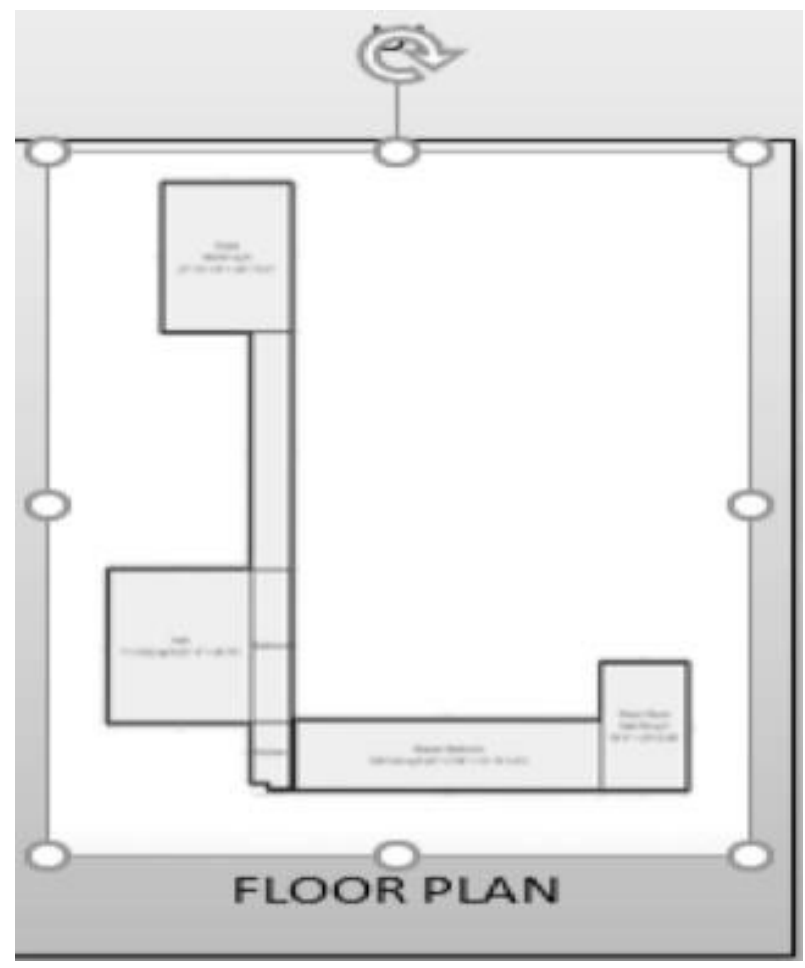

Fig. 4.Screenshot of floor plan for our site

\section{B. BLE Beacon Positioning}

Beacon is a hardware that uses low-power Bluetooth technology. It is a small signal transmitter and has applications in field like indoor navigation, mobile payment, store browsing, indoor positioning and item tracking. Each beacon will have unique UUID (Universal Unique Identifier).Incase of a multilevel building, the floor where the user is located can be identified using the UUID of the respective floor's beacon.

Published By:

Blue Eyes Intelligence Engineering

\& Sciences Publication

(C) Copyright: All rights reserved.

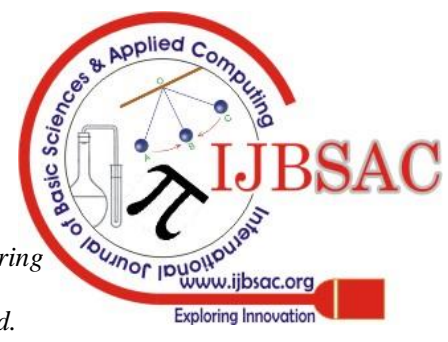


The trilateration method solves many problems related to positioning and thereby enabling the location accuracy of the computing device. The received signal strength (RSS), time of arrival (ToA) and time difference of arrival (TDoA) are the measurement techniques that are used in trilateration method. The optimal position of the device can be obtained by using this technique. The BLE beacons are arranged in trilateral position to provide better results in accuracy.

\section{*(the beacon screen shot along with its range coverage should be added)}

\section{C.Fingerprinting \& Recording waypoints and paths}

Magnetic and BLE beacon fingerprints are collected using magnetometer and other sensors of the dedicated software device using which the current location of the user can be identified. Locating the beacon's position in the floor plan (using trilateration technique), plotting waypoints along the way path in the floor plan and calibrating to record all the possible paths for the product aisle navigation in the retail.

\section{D.Map Generation and Testing}

The floor plan must be according to the scale of the retail and is uploaded to the API. We can align the floor plan either by manually specifying the geo-coordinates or by overlaying the plan on google maps. Once the map generation is done, testing can be carried out if the accuracy obtained is satisfactory.

\section{E.Building the application}

After placing the floor plan in the exact longitude and latitude values of the retail, the application will fetch you the floor plan along with current location identification(of the customer).On taping the aisle of interest, the user will be navigated. This enables the application to be location sensitive (location obtained is in WGS84 format) and context aware.

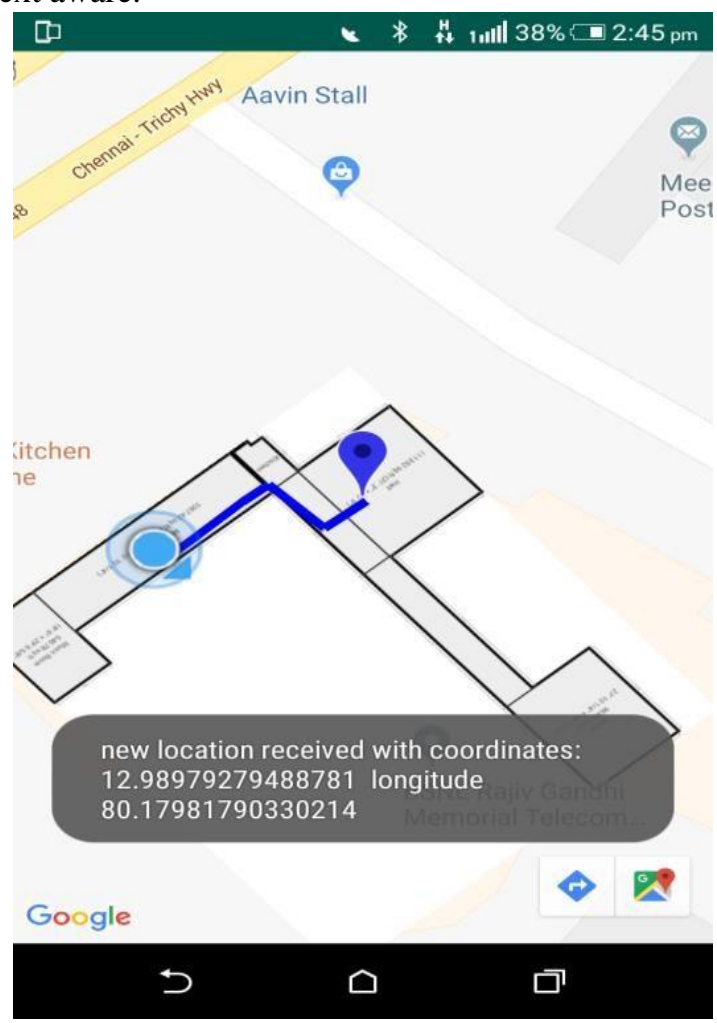

Fig. 5. Screenshot of final outcome

\section{CONCLUSION}

In today's world, smart phones play the major part in the living. These android smart phones (version 4.3 or higher) are provided with many sensors like compass, Accelero meter, gyroscope, magneto meter ,etc., hence android has been selected as a platform to develop Indoor Positioning System. These sensors are used in magnetic localization for fingerprinting and accessing the current location along with the BLE Beacons hat enables better accuracy in IPS. Experimental results rendered an android based navigation application and no infrastructure cost is evolved using magnetic localization. The application potential of indoor positioning system is immensely powerful and it can be linked with similar technology like augmented reality and Internet of Things, expanding upon its business opportunities and market potential. However, currently there are still many issues to overcome for wider application of this technology, such as optimization of the positioning (need for more fingerprinting), real-time message communication and battery life of the system.

\section{REFERENCES}

1. Adam Satan, 2018. Bluetooth based Indoor Navigation Mobile system: International Carpathian Control Conference.

2. Amit Singh, Yash Shreshthi, Neelay Waghchoure, Amey Wakchaure, 2018. Indoor Navigation System using Bluetooth Low Energy Beacons. International Conference Computing Communication Control and Automation.

3. Ding-Yu Liu, Cheng-Yu Hsieh, 2018. Study of Indoor exhibition using BEACON's Mobile Navigation. Internationa Conference on Advanced Manufacturing.

4. Taiga Arai, Takahiro Yoshizawa, Takuya Aoki, Keiichi Zempo, Ykihiko Okada, 2019. Evaluation of Indoor Positioning System based on Attachable Infrared Beacons in Metal Shelf Environment. International Conference on Consumer Electronics.

5. Andrew Mackey, PetrosSpachos, Konstantinos N, Plataniotis, 2018 Enhanced Indoor Navigation System with Beacons and Kalman Filters. Global Conference on Signal and Information Processing.

6. Stephen Statler, 2016. Beacon Technologies: The Hitchhiker's Guide of the Beaco system.

\section{AUTHORS PROFILE}

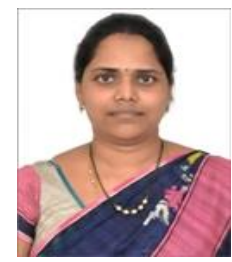

Ms. Anusha Sanampudi, B.Tech.,M.E.,(PhD), currently working as Assistant Professor in department of Computer Science and Engineering, R.M.K Engineering College, Chennai. She obtained her B.Tech (CSE) from KIET, JNTU, Hyderabad and M.E(CSE) from R.M.K Engineering College, Anna University, Chennai. Currently Pursuing Ph.D in Anna University, Chennai. She has been in teaching profession for the past 10 years. Her area of interest includes Big Data Analytics, Machine Learning, Artificial Intelligence. She has published 4 papers in international and national journals. She has attended many Workshops and FDPs sponsored by AICTE related to her area of interest. She is life time member for ISTE,CSTA,IAENG.

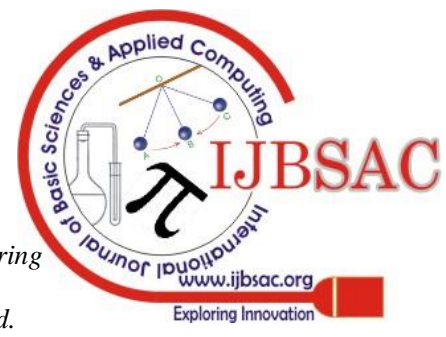

